\title{
Vector and Scalar Modes in Coherent Mode Representation of Electromagnetic Beams
}

\author{
Kisik Kim* \\ Department of Physics, Inha University, Inchon 402-751, Republic of Korea
}

(Received April 4, 2008 : revised May 27, 2008 : accepted May 28, 2008)

\begin{abstract}
It is shown that the two mode representations, one with vector modes and the other with scalar modes, for the cross spectral density matrices of electromagnetic beams are equivalent to each other. In particular, we suggest a method to find the vector modes from the scalar modes and formulate the cross spectral density matrix as a correlation matrix.
\end{abstract}

Keyword: Coherent mode representation, Coherence, Electromagnetic beams

OCIS codes : (260.5430) Polarization; (030.1640) Coherence; (000.5490) Probability theory, stochastic processes and statistics

The unified theory of coherence and polarization serves as the natural ground for the statistical theory of random electromagnetic beams. [1] Accordingly, the coherent mode representation for the scalar field is also generalized to the vector field, however, the procedure to determine vector modes becomes rather involved compared with scalar modes, and requires solving a vector integral equation. [2-3] On the other hand, a different mode representation has been introduced, which consists of two scalar coherent mode representations for the diagonal elements and one bi-modal expansion for the off-diagonal elements of the cross spectral density matrix. [4]In this paper, we consider the connection between these two mode representations. In particular, we suggest a method to find the vector modes from the scalar modes and formulate the cross spectral density matrix as a correlation matrix.

Let us consider a random electromagnetic beam propagating close to the $z$-direction. Its coherence properties and polarization properties may be expressed in terms of its $2 \times 2$ cross-spectral density matrix [1]

$$
\vec{W}\left(\vec{r}_{1}, \vec{r}_{2}, \omega\right) \equiv\left[W_{i j}\left(\vec{r}_{1}, \vec{r}_{2}, \omega\right)\right]=\left\lfloor\left\langle E_{i}^{*}\left(\vec{r}_{1}, \omega\right) E_{j}\left(\vec{r}_{2}, \omega\right)\right\rangle\right] .
$$

Here, $E_{i}$ and $E_{j}$ are components of a typical realization of a monochromatic electric field of frequency $\omega$ along two mutually orthogonal directions perpendicular to the $z$-direction, asterisk denotes the complex conjugate and the sharp brackets denote ensemble average, in the sense of coherence theory in the space-frequency domain [5].

The coherent mode representation for a scalar field may be extended to an electromagnetic beam and the cross spectral density matrix can be expanded as an absolutely and uniformly convergent Mercer-type series [2]

$$
\vec{W}\left(\vec{r}_{1}, \vec{r}_{2}, \omega\right)=\sum_{k=1}^{K} \lambda_{k}(\omega) \vec{E}_{k}^{*}\left(\vec{r}_{1}, \omega\right) \vec{E}_{k}^{T}\left(\vec{r}_{2}, \omega\right),
$$

where $\lambda_{k}(\omega)$ and $\vec{E}_{k}(\vec{r}, \omega)=\left[E_{i, k}(\vec{r}, \omega)\right]$ are the eigenvalues and eigenvectors of the Fredholm integral equation

$$
\int_{D} \vec{E}_{k}^{T}\left(\vec{r}_{1}, \omega\right) \overleftrightarrow{W}\left(\vec{r}_{1}, \vec{r}_{2}, \omega\right) d^{2} r_{1}=\lambda_{k}(\omega) \vec{E}_{k}^{T}\left(\vec{r}_{2}, \omega\right),
$$

where the integration is carried out over the domain $D$. This coherent mode expansion is similar to the KarhunenLoeve expansion for a complex random process [6]. The eigenvalues are nonnegative and the eigenfunctions satisfy the orthogonality condition

$$
\int_{D} \vec{E}_{k}^{T}(\vec{r}, \omega) \vec{E}_{l}^{*}(\vec{r}, \omega) d^{2} r=\delta_{k l}
$$

*Corresponding author: kisik@inha.ac.kr 
For our convenience, we write the two-component vector $\vec{E}_{k}(\vec{r}, \omega)$ in the form of a column matrix, i.e.,

$$
\vec{E}_{k}(\vec{r}, \omega)=\left(\begin{array}{c}
E_{x, k}(\vec{r}, \omega) \\
E_{y, k}(\vec{r}, \omega)
\end{array}\right),
$$

and consequently

$$
\vec{E}_{k}^{T}(\vec{r}, \omega)=\left(E_{x, k}(\vec{r}, \omega), \quad E_{y, k}(\vec{r}, \omega)\right) .
$$

Once the vector coherent mode representation is obtained, the ensemble realization for the given cross spectral density matrix is immediately accomplished and the corresponding ensemble may be expressed by

$$
\left\{\vec{E}\left(\vec{r}, \omega ; a_{1}, a_{2}, \cdots, a_{K}\right)\right\}=\left\{\sum_{k=1}^{K} a_{k} \vec{E}_{k}(\vec{r}, \omega)\right\},
$$

where the stochastic variables $a_{k}$ satisfy

$$
\left\langle a_{k}^{*} a_{l}\right\rangle=\lambda_{k}(\omega) \delta_{k l} .
$$

Instead of the vector coherent mode representation, the scalar mode representations have been used to describe the stochastic, planar, electromagnetic sources [4]. The diagonal elements of the cross spectral density matrix have coherent mode representations which may be written explicitly as

$$
W_{x x}\left(\vec{r}_{1}, \vec{r}_{2}, \omega\right)=\sum_{n=1}^{N} \lambda_{n}^{(x)}(\omega) \phi_{n}^{*}\left(\vec{r}_{1}, \omega\right) \phi_{n}\left(\vec{r}_{2}, \omega\right)
$$

and

$$
W_{y y}\left(\vec{r}_{1}, \vec{r}_{2}, \omega\right)=\sum_{m=1}^{M} \lambda_{m}^{(y)}(\omega) \psi_{m}^{*}\left(\vec{r}_{1}, \omega\right) \psi_{m}\left(\vec{r}_{2}, \omega\right),
$$

where the eigenvalues and the eigenfunctions satisfy the following integral eigenvalue equations:

$$
\begin{aligned}
& \int_{D} W_{x x}\left(\vec{r}_{1}, \vec{r}_{2}, \omega\right) \phi_{n}\left(\vec{r}_{1}, \omega\right) d^{2} r_{1}=\lambda_{n}^{(x)}(\omega) \phi_{n}\left(\vec{r}_{2}, \omega\right), \\
& \int_{D} W_{y y}\left(\vec{r}_{1}, \vec{r}_{2}, \omega\right) \psi_{m}\left(\vec{r}_{1}, \omega\right) d^{2} r_{1}=\lambda_{m}^{(y)}(\omega) \psi_{m}\left(\vec{r}_{2}, \omega\right) .
\end{aligned}
$$

The off-diagonal elements of the cross spectral density matrix do not have coherent mode representations, however, they may possess bi-modal expansions given by

$$
\begin{aligned}
& W_{x y}\left(\vec{r}_{1}, \vec{r}_{2}, \omega\right)=\sum_{n=1}^{N} \sum_{m=1}^{M} \Lambda_{n m}(\omega) \phi_{n}^{*}\left(\vec{r}_{1}, \omega\right) \psi_{m}\left(\vec{r}_{2}, \omega\right), \\
& W_{y x}\left(\vec{r}_{1}, \vec{r}_{2}, \omega\right)=\sum_{n=1}^{N} \sum_{m=1}^{M} \Lambda_{n m}^{*}(\omega) \psi_{m}^{*}\left(\vec{r}_{1}, \omega\right) \phi_{n}\left(\vec{r}_{2}, \omega\right),
\end{aligned}
$$

where the coefficients $\Lambda_{n m}(\omega)$ are obtained by

$$
\Lambda_{n m}(\omega)=\int_{D} d^{2} r_{1} \int_{D} d^{2} r_{2} \phi_{n}\left(\vec{r}_{1}, \omega\right) W_{x y}\left(\vec{r}_{1}, \vec{r}_{2}, \omega\right) \psi_{m}^{*}\left(\vec{r}_{2}, \omega\right) .
$$

We may realize an ensemble of the electromagnetic beam in terms of two sets of coherent mode functions $\left\{\phi_{n}(\vec{r}, \omega)\right\}$ and $\left\{\psi_{m}(\vec{r}, \omega)\right\}$. We write the electric field vector as

$$
\vec{E}(\vec{r}, \omega)=\left(\begin{array}{c}
\sum_{n=1}^{N} a_{n} \phi_{n}(\vec{r}, \omega) \\
\sum_{m=1}^{M} b_{m} \psi_{m}(\vec{r}, \omega)
\end{array}\right),
$$

where $a_{n}$ and $b_{m}$ are random complex coefficients. We can construct a correlation matrix from the ensemble given by Eq. (16) as

$$
\begin{aligned}
& W_{x x}\left(\vec{r}_{1}, \vec{r}_{2}, \omega\right)=\sum_{n=1}^{N} \sum_{n^{\prime}=1}^{N}\left\langle a_{n}^{*} a_{n^{\prime}}\right\rangle \phi_{n}^{*}\left(\vec{r}_{1}, \omega\right) \phi_{n^{\prime}}\left(\vec{r}_{2}, \omega\right), \\
& W_{y y}\left(\vec{r}_{1}, \vec{r}_{2}, \omega\right)=\sum_{m=1}^{M} \sum_{m^{\prime}=1}^{M}\left\langle b_{m}^{*} b_{m^{\prime}}\right) \psi_{m}^{*}\left(\vec{r}_{1}, \omega\right) \psi_{m^{\prime}}\left(\vec{r}_{2}, \omega\right), \\
& W_{x y}\left(\vec{r}_{1}, \vec{r}_{2}, \omega\right)=\sum_{n=1}^{N} \sum_{m=1}^{M}\left\langle a_{n}^{*} b_{m}\right\rangle \phi_{n}^{*}\left(\vec{r}_{1}, \omega\right) \psi_{m}\left(\vec{r}_{2}, \omega\right)
\end{aligned}
$$

On comparing Eqs. (9), (10), and (13) with Eqs. (17), (18), and (19), we require the random coefficients to satisfy

$$
\begin{aligned}
& \left\langle a_{n}^{*} a_{n^{\prime}}\right\rangle=\lambda_{n}^{(x)} \delta_{n n^{\prime}}, \\
& \left\langle b_{m}^{*} b_{m^{\prime}}\right\rangle=\lambda_{m}^{(y)} \delta_{m m^{\prime}}, \\
& \left\langle a_{n}^{*} b_{m}\right\rangle=\Lambda_{n m} .
\end{aligned}
$$

The equations (20), (21), and (22) imply that $\left\{a_{n}\right\}$ and $\left\{b_{m}\right\}$ are uncorrelated within themselves, however, there exist cross-correlations between $\left\{a_{n}\right\}$ and $\left\{b_{m}\right\}$. We then raise a question whether there exist $(N+M)$ random coefficients satisfying the correlations given by Eqs. (20), (21), and (22). In other words, can we find a new set of random coefficients, say $\left\{C_{n}\right\}$, which are completely uncorrelated? Once we find such random coefficients, an ensemble realization can be obtained by simply taking $c_{n}=\sqrt{\lambda_{n}} e^{i \theta_{n}}$, where $\lambda_{n}$ is the strength of 
the corresponding mode and $\theta_{n}$ is a randomly distributed real number in $[0,2 \pi)$. The answer to this question is "yes" and it turns out that finding a new set of uncorrelated coefficients is equivalent to constructing the vector modes from two coherent mode representations and a bi-modal expansion. The existence of those random coefficients may be proved by constructing the vector modes explicitly out of the scalar modes as below.

We now show that two coherent mode representations for the diagonal elements and two bi-modal expansions for the off-diagonal elements of the cross spectral density matrix uniquely determine the vector coherent mode representation of the cross spectral density matrix. For this purpose, we write an arbitrary electric field vector of the electromagnetic beam in terms of the mode functions $\left\{\phi_{n}(\vec{r}, \omega)\right\}$ and $\left\{\psi_{m}(\vec{r}, \omega)\right\}$

$$
\vec{E}(\vec{r}, \omega)=\left(\begin{array}{c}
\sum_{n=1}^{N} \alpha_{n} \phi_{n}(\vec{r}, \omega) \\
\sum_{m=1}^{M} \beta_{m} \psi_{m}(\vec{r}, \omega)
\end{array}\right),
$$

where $\left\{\alpha_{n}\right\}$ and $\left\{\beta_{m}\right\}$ are complex numbers, in general. We now calculate

$$
I(\vec{E}) \equiv \int_{D} d^{2} r_{1} \int_{D} d^{2} r_{2} \vec{E}^{T}\left(\vec{r}_{1}, \omega\right) \vec{W}\left(\vec{r}_{1}, \vec{r}_{2}, \omega\right) \vec{E}^{*}\left(\vec{r}_{2}, \omega\right) .
$$

On using two coherent mode representations and two bi-modal expansions for elements of the cross spectral density matrix, after a straightforward calculation, we obtain the following expression of $I(\vec{E})$ :

$$
I(\vec{E})=\sum_{n=1}^{N} \lambda_{n}^{(x)} \alpha_{n}^{*} \alpha_{n}+\sum_{n=1}^{N} \sum_{m=1}^{M} \Lambda_{m n} \alpha_{n}^{*} \beta_{m}+\sum_{n=1}^{N} \sum_{m=1}^{M} \Lambda_{n m} \alpha_{n} \beta_{m}^{*}+\sum_{m=1}^{M} \lambda_{m}^{(y)} \beta_{m}^{*} \beta_{m} .
$$

This expression can be expressed in the matrix form as

$$
I(\vec{E})=\vec{A}^{T} \vec{\Omega} \vec{A}^{*},
$$

where

$$
\begin{aligned}
\vec{A} & =\left(\begin{array}{l}
\vec{\alpha} \\
\vec{\beta}
\end{array}\right), \\
\vec{\Omega} & =\left(\begin{array}{cc}
\vec{L}^{(x)} & \vec{\Lambda} \\
\vec{\Lambda}^{+} & \vec{L}^{(y)}
\end{array}\right),
\end{aligned}
$$

$$
\begin{aligned}
& \vec{\alpha}=\left(\begin{array}{c}
\alpha_{1} \\
\alpha_{2} \\
\cdot \\
\cdot \\
\alpha_{N}
\end{array}\right), \quad \vec{\beta}=\left(\begin{array}{c}
\beta_{1} \\
\beta_{2} \\
\cdot \\
\cdot \\
\beta_{M}
\end{array}\right), \\
& \widetilde{L}^{(x)}=\left(\begin{array}{ccccc}
\lambda_{1}^{(x)} & 0 & \cdot & \cdot & 0 \\
0 & \lambda_{2}^{(x)} & 0 & \cdot & \cdot \\
\cdot & 0 & \cdot & \cdot & \cdot \\
\cdot & \cdot & \cdot & \cdot & 0 \\
0 & \cdot & \cdot & 0 & \lambda_{N}^{(x)}
\end{array}\right), \\
& \vec{L}^{(y)}=\left(\begin{array}{ccccc}
\lambda_{1}^{(y)} & 0 & \cdot & \cdot & 0 \\
0 & \lambda_{2}^{(y)} & 0 & \cdot & \cdot \\
\cdot & 0 & \cdot & \cdot & \cdot \\
\cdot & \cdot & \cdot & \cdot & 0 \\
0 & \cdot & \cdot & 0 & \lambda_{M}^{(y)}
\end{array}\right), \\
& \vec{\Lambda}=\left(\begin{array}{ccccc}
\Lambda_{11} & \Lambda_{12} & \cdot & \cdot & \Lambda_{1 M} \\
\Lambda_{21} & \Lambda_{22} & \cdot & \cdot & \cdot \\
\cdot & \cdot & \cdot & \cdot & \cdot \\
\cdot & \cdot & \cdot & \cdot & \cdot \\
\Lambda_{N 1} & \cdot & \cdot & \cdot & \Lambda_{N M}
\end{array}\right) .
\end{aligned}
$$

The rank of the matrix $\vec{\Omega}$ is $(N+M)$ and it is Hermitian. The non-negative definite character of the cross spectral density matrix is now transferred to the matrix $\vec{\Omega}$. It is evident that $\vec{\Omega}$ has $(N+M)$ eigenvectors and the corresponding eigenvalues and the ensemble realization can be obtained exactly same way as in Eqs. (7) and (8). For elucidating the procedure to get vector modes from scalar modes explicitly, we present one simple example below.

We consider each diagonal element has only one mode respectively and the corresponding coherent mode representations are given by

$$
W_{x x}\left(\vec{r}_{1}, \vec{r}_{2}, \omega\right)=\lambda(\omega) \phi^{*}\left(\vec{r}_{1}, \omega\right) \phi\left(\vec{r}_{2}, \omega\right),
$$

and

$$
W_{y y}\left(\vec{r}_{1}, \vec{r}_{2}, \omega\right)=\lambda(\omega) \psi^{*}\left(\vec{r}_{1}, \omega\right) \psi\left(\vec{r}_{2}, \omega\right),
$$

where we set $\lambda^{(x)}=\lambda^{(y)} \equiv \lambda$ for convenience. The offdiagonal elements of the cross spectral density matrix should necessarily be written as

$$
\begin{aligned}
& W_{x y}\left(\vec{r}_{1}, \vec{r}_{2}, \omega\right)=\Lambda(\omega) \phi^{*}\left(\vec{r}_{1}, \omega\right) \psi\left(\vec{r}_{2}, \omega\right), \\
& W_{y x}\left(\vec{r}_{1}, \vec{r}_{2}, \omega\right)=\Lambda^{*}(\omega) \psi^{*}\left(\vec{r}_{1}, \omega\right) \phi\left(\vec{r}_{2}, \omega\right) .
\end{aligned}
$$


The corresponding matrix $\ddot{\Omega}$ may be written as

$$
\vec{\Omega}=\left(\begin{array}{cc}
\lambda & \Lambda \\
\Lambda^{*} & \lambda
\end{array}\right),
$$

and the eigenvalues are found to be

$$
\lambda_{ \pm}=\lambda \pm|\Lambda|
$$

The corresponding eigenvectors are also easily obtained as

$$
\vec{A}_{ \pm}=\frac{1}{\sqrt{2}}\left(\begin{array}{c}
1 \\
\pm|\Lambda| / \Lambda
\end{array}\right)
$$

and finally the two vector modes are given by

$$
\vec{E}_{ \pm}(\vec{r}, \omega)=\frac{1}{\sqrt{2}}\left(\begin{array}{c}
\phi(\vec{r}, \omega) \\
\pm\left(|\Lambda| / \Lambda^{*}\right) \psi(\vec{r}, \omega)
\end{array}\right) .
$$

When $\Lambda=0, \vec{\Omega}$ becomes diagonal and two eigenvalues are the same. Any two mutually orthogonal vectors can be taken as the corresponding eigenvectors and the simplest ones may be

$$
\vec{A}_{1}=\left(\begin{array}{l}
1 \\
0
\end{array}\right), \quad \vec{A}_{2}=\left(\begin{array}{l}
0 \\
1
\end{array}\right) .
$$

The two vector modes are then given by

$$
\vec{E}_{1}(\vec{r}, \omega)=\left(\begin{array}{c}
\phi(\vec{r}, \omega) \\
0
\end{array}\right), \quad \vec{E}_{2}(\vec{r}, \omega)=\left(\begin{array}{c}
0 \\
\psi(\vec{r}, \omega)
\end{array}\right)
$$

In this paper, we considered the connection between the vector mode representation for an entire cross spectral density matrix and the two scalar mode representations for two diagonal matrix elements of the cross spectral density matrix of an electromagnetic beam. We explicitly constructed the vector modes out of the two kinds of scalar modes and showed that the number of vector modes is given by the sum of two numbers of scalar modes. These results have been demonstrated through simple examples.

\section{ACKNOWLEDGEMENT}

This work was supported by Inha University.

\section{REFERENCES}

[1] E. Wolf, Phys. Letts. A312, 263, 2003.

[2] F. Gori, M. Santarsiero, R. Simon, G. Piquerro, R. Borghi, and G. Guattari, JOSA A 20, 78, 2003.

[3] J. Tervo, T. Setälä and A. T. Friberg, JOSA A21, $2205,2004$.

[4] K. Kim and E. Wolf, Opt. Commun. 261, 19, 2006.

[5] L. Mandel and E. Wolf, Optical Coherence and Quantum Optics(Cambridge, Cambridge University Press, 1995), Sec. 4.7.

[6] L. Mandel and E. Wolf, Optical Coherence and Quantum Optics(Cambridge, Cambridge University Press, 1995), Sec. 2.5. 